ARTICLE

Received 12 Aug 2014 | Accepted 17 Oct 2014 | Published 3 Dec $2014 \quad$ DOl: 10.1038/ncomms6597

\title{
Photochemical formation of intricarene
}

\author{
Desiree Stichnoth ${ }^{1,2, \star}$, Patrick Kölle ${ }^{1, \star}$, Thomas J. Kimbrough ${ }^{1,2}$, Eberhard Riedle ${ }^{3}$, Regina de Vivie-Riedle ${ }^{1}$ \\ \& Dirk Trauner ${ }^{1,2}$
}

Sunlight is the ultimate driver of biosynthesis but photochemical steps late in biosynthetic pathways are very rare. They appear to play a role in the formation of certain furanocembranoids isolated from Caribbean corals. One of these compounds, intricarene, has been suspected to arise from an intramolecular 1,3-dipolar cycloaddition involving an oxidopyrylium. Here we show, by a combination of experiments and theory, that the oxidopyrylium forms under photochemical conditions and that its cycloaddition occurs via a triplet state. The formation of a complex by-product can be rationalized by another photochemical step that involves a conical intersection. Our work raises the question whether intricarene is biosynthesized in the natural habitat of the corals or is an artefact formed during workup. It also demonstrates that the determination of exact irradiation spectra, in combination with quantum chemical calculations, enables the rationalization of complex reaction pathways that involve multiple excited states.

\footnotetext{
${ }^{1}$ Department of Chemistry, Ludwig-Maximilians-Universität, 81377 Munich, Germany. ${ }^{2}$ Center for Integrated Protein Science, 81377 Munich, Germany.

${ }^{3}$ Faculty of Physics, Lehrstuhl für BioMolekulare Optik, Ludwig-Maximilians-Universität, 80538 Munich, Germany. * These authors contributed equally to this work. Correspondence and requests for materials should be addressed to R.de.V.-R. (email: Regina.de_Vivie@cup.uni-muenchen.de) or to D.T. (email: dirk.trauner@lmu.de).
} 
$\mathrm{P}$ hotosynthesis involves the interconversion of light energy into chemical energy, which then drives the complex pathways of secondary metabolism. Photochemical reactions that occur late in a biosynthesis, however, are very rare. In addition, it is often debatable whether they are genuine biosynthetic steps or they take place when biological materials get processed and stored in light. The former seems to apply to the photochemical formation of cholecalciferol (vitamin $\mathrm{D}_{3}$ ) from 7-dehydrocholesterol. Although the majority of cholecalciferol is taken up from dietary sources, it has been suggested that a lack of sunlight is responsible for the development of rickets and other diseases associated with low levels of vitamin $\mathrm{D}_{3}$ (ref. 1).

As with the aetiology of rickets, the geographical latitude and solar irradiance have to be taken into account when the biological significance of photochemical reactions is considered. Situated at the Tropic of Cancer, the Caribbean belongs to the most intensely irradiated regions of the earth. Therefore, it is not surprising that several natural products have been isolated from the Caribbean whose formation seems to require light. The photochemical isomerization of bipinnatin J (1) to kallolide A (2) is a case in point (Fig. 1) ${ }^{2}$. Both furanocembranoids have been isolated from gorgonian octocorals, which live at various depths $(1-45 \mathrm{~m})$ in the Caribbean Sea and can get exposed to relatively intense sunlight ${ }^{2,3}$. It is therefore conceivable that this photochemical reaction, which can be reproduced in high yield in the laboratory ${ }^{2,4}$, also occurs in the native environment of the animals.

Bielschowskysin (3) (ref. 5) and intricarene (4) (ref. 6), both isolated from the octocoral Pseudopterogorgia kallos, are two other natural products that have attracted much interest by the synthetic community ${ }^{7-15}$. According to a biosynthetic hypothesis that has been explored by biomimetic total synthesis, intricarene appears to stem from bipinnatin J (1) as well (Fig. 2 (refs 7,8)). Oxidation of 1 with photochemically generated singlet oxygen, followed by hydrolysis and rearrangement would yield hydroxy pyrenone 5. A formal 1,3-elimination of water would then produce an oxidopyrylium $\mathbf{6}$, whose diastereoselective transannular 1,3-dipolar cycloaddition would afford intricarene (4) in a final, concerted step. As such, intricarene could be one of the few natural products whose biosynthesis involves a 1,3dipolar cycloaddition.

Tantillo et al. ${ }^{16}$ have provided computational evidence that the key cycloaddition step is thermally feasible. According to their

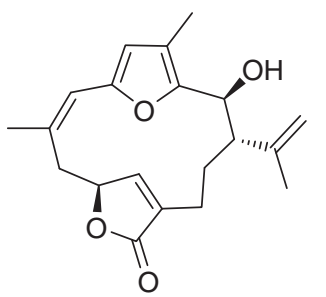

Bipinnatin J (1)

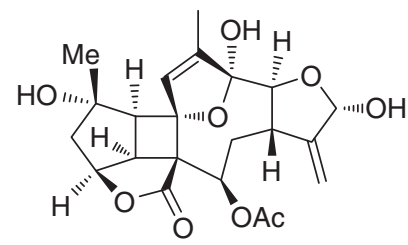

Bielschowskysin (3)

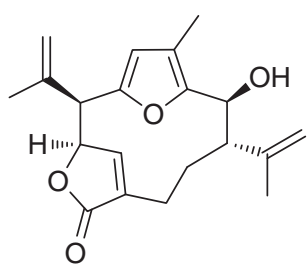

Kallolide A (2)

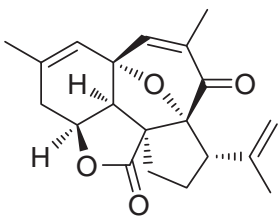

Intricarene (4)
Figure 1 | Photochemically formed natural products. The natural products relevant to the present work are shown. report, however, the calculated activation barrier of $\sim 20 \mathrm{kcal} \mathrm{mol}^{-1}$ (both in nonpolar and polar environments) appears to be relatively high, albeit still within the bounds of a reasonably fast thermal process. Reflecting this value, the published syntheses of intricarene require temperatures and conditions that certainly cannot be deemed 'biomimetic' (refluxing DMSO or DMF) and the yields obtained were generally poor $^{7,8}$. In addition to this, the exact biosynthetic pathway that would lead from the Achmatowicz oxidation product 5 to the high-energy oxidopyrylium 6 remained unclear.

We now show that intricarene can be formed photochemically from a derivative of bipinnatin $J$ (1) under conditions that resemble those found in the Caribbean. In the course of our studies, we have identified a structurally intriguing by-product that may well be found in extracts from P. bipinnata too. To interpret our results, we have carried out ab initio calculations on the photochemical formation of the oxidopyrylium species and their reactions in the excited state that provide new insights into the chemistry of these useful intermediates.

\section{Results}

Photochemical synthesis of intricarene. Our experimental results were obtained serendipitously during a model study directed at bielschowskysin, the formation of which could also involve photochemistry (Fig. 3 and Supplementary Discussion). Treatment of racemic bipinnatin J (1), obtained by total synthesis ${ }^{17}$, with methanol under slightly acidic conditions gave O-Methyl bipinnatin J (7). The latter has been isolated before, in enantiomerically pure form, from a methanolic extract of Pseudopterogorgia bipinnata ${ }^{18}$. Oxidation of 7 with singlet oxygen yielded the sensitive diene dione $\mathbf{8}$, which, being an ether, cannot undergo acetal formation. Structurally, it resembles the known diene-dione natural products coralloidolide $\mathrm{E}$ and isoepilophodione $\mathrm{B}^{19,20}$. We reasoned that conjugate attack of a molecule of water at $\mathrm{C} 8$ of $\mathbf{8}$ could give rise to enol acetal $\mathbf{9}$, which bears the requisite functional groups for a photochemical $[2+2]$ cycloaddition. If successful, this reaction would yield cyclobutane derivative 10, a close congener of bielschowskysin.

To mimic Caribbean sunlight, we decided to use a reptile lamp, a type of light source that is known for its significant UV content. Irradiation of 8 in a 1:1 mixture of deuterated acetone and water with a commercially available reptile lamp (nominal power consumption of $275 \mathrm{~W}$ ) yielded two isolable products. To our considerable surprise, one of them was intricarene (4), isolated with a yield of $25 \%$, whereas the other one was hydroxy cyclopentenone 11, which was isolated with a yield of $15 \%$. The $\mathrm{X}$-ray structures of racemic compounds $\mathbf{4}$ and $\mathbf{1 1}$ are shown in Fig. 4. Structural data of racemic $\mathbf{4}$ and $\mathbf{1 1}$ have been deposited

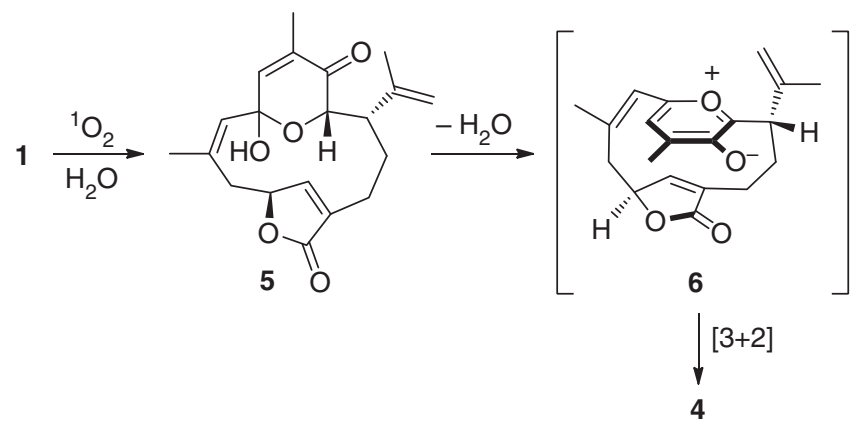

Figure 2 | Biosynthetic relationship of bipinnatin $\mathbf{J}$ and intricarene. Achmatowizc-type oxidation of bipinnatin J, followed by dehydration and 1,3-dipolar cycloaddition was proposed to account for the formation of intricarene. 
a

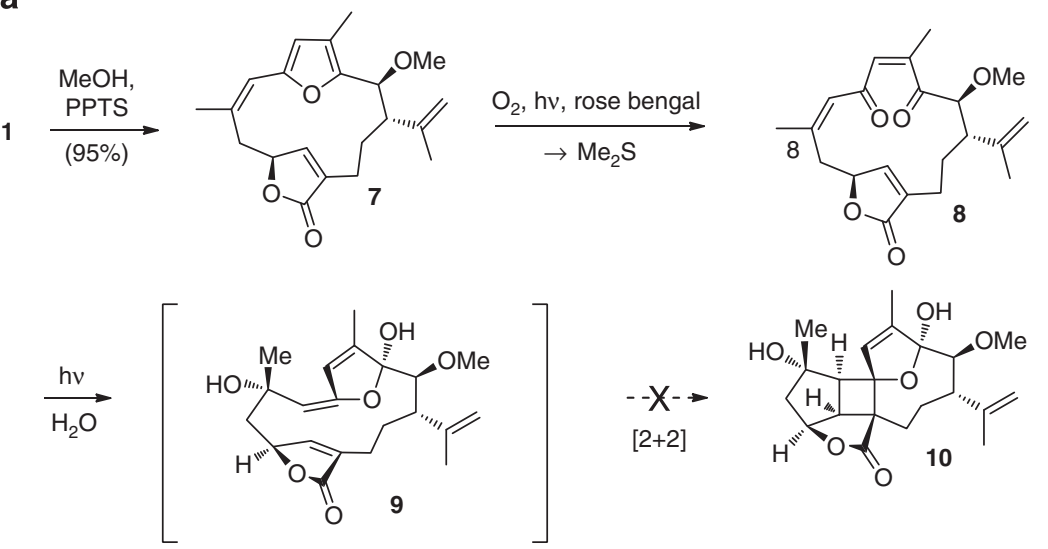

b

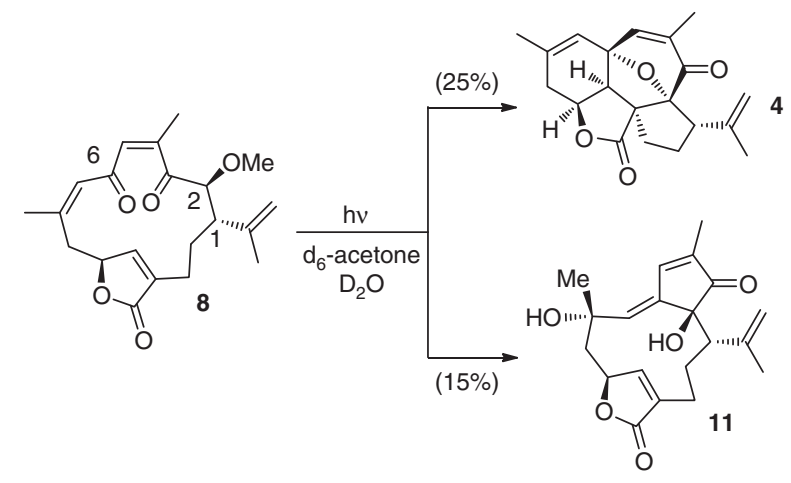

C

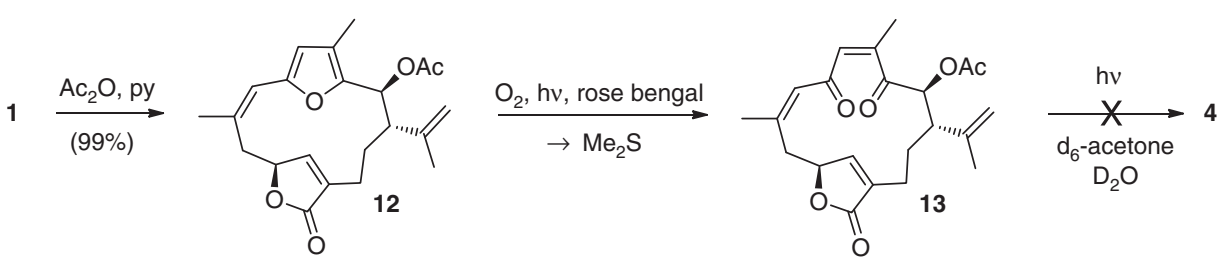

Figure 3 | Overview of the synthetic route from bipinnatin $\mathbf{J}(\mathbf{1})$ to intricarene (4) and hydroxy cyclopentenone 11. (a) Attempted synthesis of the bielschowskysin skeleton via conjugate addition of water and photochemical interception of the presumed intermediate. Conversion of bipinnatin J (1) to its methyl ether $\mathbf{8}$ via $S_{N} 1$ reaction was followed by oxidative cleavage of the furan ring to yield diene dione $\mathbf{8}$. Conjugate addition of water was supposed to yield $\mathbf{9}$ and enable a photochemical [2+2] cycloaddition to form the central four-membered ring of bielschowskysin. (b) Unexpected formation of intricarene (4) and hydroxy cyclopentenone $\mathbf{1 1}$ under photochemical conditions. (c) Synthesis of acetate $\mathbf{1 2}$ and diene-dione $\mathbf{1 3}$ and failure to yield intricarene under identical photochemical conditions.
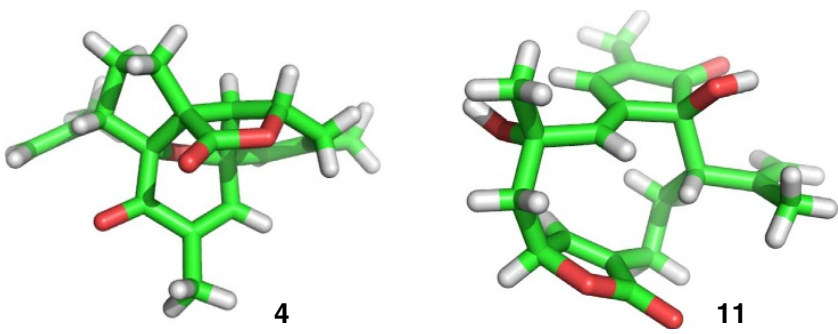

Figure 4 | X-ray structures of the final products. X-ray structure of racemic intricarene (4) and hydroxy cyclopentenone $\mathbf{1 1 .}$

with the Cambridge Crystallographic Data Centre (CCDC accession numbers 1026998 and 1026997, respectively). The photochemical procedure was highly reproducible provided the reptile lamp was used. Notably, it failed when other substrates, such as acetal $\mathbf{5}$ and acetate 13, were employed.
Identification of the photo-induced reaction path. Our experimental results raise interesting mechanistic and biosynthetic questions, which we decided to address with photophysical considerations and quantum chemical calculations at the CASSCF/ CASPT2 and DFT/TDDFT level of theory ${ }^{21}$. Solvation effects (water) were incorporated implicitly by single-point calculations of the optimized gas phase geometries using the state-specific polarizable continuum model (PCM, see Supplementary Table 4). As intersystem crossing plays a key role in photochemistry ${ }^{22,23}$ and intermediate triplet states can be involved in light-induced cycloadditions ${ }^{24,25}$, we investigated the singlet as well as the triplet states of the initially excited 8. As a first step, we measured the emission spectrum of the reptile lamp to compare it with the calculated and experimentally determined absorption spectrum of our starting materials and postulated key intermediates. As can be seen in Fig. 5, the lamp spectrum has sharp lines in the visible and $\mathrm{UV}-\mathrm{A}$ region of the electromagnetic spectrum and only negligible contributions below 360 or above $700 \mathrm{~nm}$. The peak emissions at $\lambda_{\max }=366,405,436,492,546,578$ and $679 \mathrm{~nm}$ can all be 


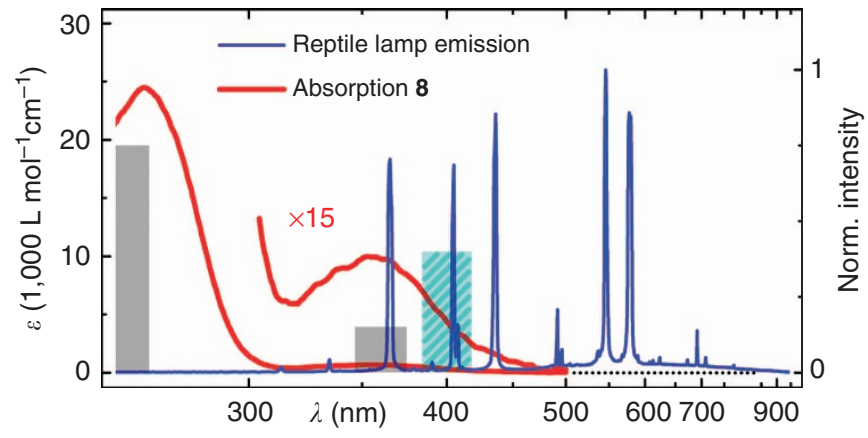

Figure 5 | Comparison of the precursor absorption spectrum with the illumination spectrum and the calculated transitions. Emission spectrum of the reptile lamp (blue). Absorption of $\mathbf{8}$ (red) and calculated transitions for $\mathbf{8}$ (grey bars) and $\mathbf{6}$ (cyan bar).

assigned to $\mathrm{Hg}$ emissions. This means that UV-B light is virtually absent in this type of lamp, presumably due to the type of glass used for the outer bulb. To determine the irradiance applied in the photochemical synthesis, we determined the light intensity at a distance of $15 \mathrm{~cm}$ in front of the lamp (see Supplementary Discussion). This geometry corresponds to the synthetic conditions. Standard chemical actinometry proved applicable only to a limited extent due to the high intensity of the lamp and the constrained geometry of the NMR tube used as vessel. It therefore provides only a lower boundary. For comparison photometry was also employed that provides an upper boundary for the irradiance.

The experimentally determined absorption spectrum of diene dione 8 shows a weak absorption band in the deep blue and UV-A region around $\lambda_{\max }=360 \mathrm{~nm}$ and strong absorption around $\lambda_{\max }=265 \mathrm{~nm}$ (Fig. 5). Only the weak absorption band $(360 \mathrm{~nm})$ matches with the emission of our reptile lamp. The photo-initiation of the conversion of $\mathbf{8}$ therefore has to proceed through the $360 \mathrm{~nm}$ transition.

In good agreement with the experiment, calculations reveal two absorption bands in the spectral region of interest (grey bars in Fig. 5; see Supplementary Methods (quantum chemical calculations) and Supplementary Table 1 and Fig. 3 for details). The most intense band is attributed to an intense $\pi \pi^{*}$ transition from the $S_{0}$ to the $S_{4}$ state at $260 \mathrm{~nm}$ with partial charge transfer character. As the reptile lamp used does not emit in the UV-B region, however, it is irrelevant and we focus on the $360-\mathrm{nm}$ band.

For the synthesis the solution of $\mathbf{8}$ was contained in a standard NMR tube with $4.2 \mathrm{~mm}$ inner diameter. We determined the maximum of the molar absorption coefficient of 8 to $\varepsilon_{265} \mathrm{~nm}=24,500 \mathrm{lmol}^{-1} \mathrm{~cm}^{-1}$. This renders a value of $6701 \mathrm{~mol}^{-1} \mathrm{~cm}^{-1}$ for $\varepsilon_{360} \mathrm{~nm}$ and a penetration depth of the UV-A light on the order of $1 \mathrm{~mm}$ (see Supplementary Fig. 1). As a consequence, certainly all of the light that spectrally overlaps with the $360 \mathrm{~nm}$ band (the strong lines at 366, 405 and $436 \mathrm{~nm}$ ) and penetrates the reaction vessel is absorbed by the bulk sample. The total number of absorbed photons during the $8 \mathrm{~h}$ irradiation time is $>2 \times 10^{21}$ according to the actinometry and $<1.2 \times 10^{22}$ according to the photometry. This photon number has to be compared with the total of $1.7 \times 10^{19}$ molecules of $\mathbf{8}$ in the $1 \mathrm{ml}$ sample with a concentration of $28 \mathrm{mmoll}^{-1}$. Nominally, this means that each molecule would be excited on average $>120$ times and $<700$ times. Even if the photochemical quantum yield is as low as 0.01 due to an efficient photophysical return path to the ground state of $\mathbf{8}$, practically all molecules will undergo the photochemical conversion in accord with the experimental observation. The long irradiation time ensures that the molecules are sufficiently exchanged by diffusion or most likely convection.
Quantum chemical analysis of the reaction path. Our rationalization of the photochemical formation of intricarene (4) and its by-product 11 from diene dione $\mathbf{8}$ is shown in Fig. 6. Irradiation of $\mathbf{8}$ with UV-A light initially populates the excited singlet state $\mathbf{8}\left(S_{1}\right)$. From the quantum chemical calculations, we find that at the Franck-Condon region the $S_{1}$ and the $T_{4}$ state of $\mathbf{8}$ are degenerate (Fig. 6a). Their spin-orbit coupling has a reasonable size of about $30 \mathrm{~cm}^{-1}$, consequently effective sub-ps intersystem crossing (ISC) can occur ${ }^{26}$. The resulting excited triplet state $\mathbf{8}\left(\mathrm{T}_{4}\right)$ then crosses the transition state TS1 $\left(\mathrm{T}_{1}\right)$, which describes the homolytic bond cleavage of the methoxy group, to yield a triplet diradical $\mathbf{1 4}\left(\mathrm{T}_{1}\right)$. This step corresponds to a photochemical cleavage of an $\alpha$-carbon-heteroatom bond, a well-known process in carbonyl photochemistry ${ }^{27}$. In case the barrier is not passed and the system relaxes back to the ground state, it can be reexcited and has again the possibility to cross the barrier with a finite probability. The large number of excitation probabilities for each molecule (see above) should allow the system to effectively overcome this barrier. In case no ISC takes place, the system will relax to the $S_{1}$ state minimum as high-energy barriers hinder the bond cleavage reaction in the singlet state. In the vicinity of the $S_{1}$ minimum a $S_{1} / S_{0}$ conical intersection seam is reached leading the system again back to the ground state (see Supplementary Table 28).

Once formed, the triplet diradical $\mathbf{1 4}\left(\mathrm{T}_{1}\right)$ undergoes hydrogen transfer via the transition state $\mathrm{TS} 2\left(\mathrm{~T}_{1}\right)$ to yield triplet oxidopyrylium $6\left(\mathrm{~T}_{1}\right)$ and methanol. According to our calculations, the oxidopyrylium $\mathbf{6}\left(\mathrm{T}_{1}\right)$ in its triplet state has two possible fates: initial bond formation between $\mathrm{C} 6$ and $\mathrm{C} 11$, via transition state TS3 $\left(\mathrm{T}_{1}\right)$, yields triplet diradical $\mathbf{1 5}\left(\mathrm{T}_{1}\right)$, which undergoes intersystem crossing and a second bond formation to furnish intricarene (4) (pathway (1). Overall, this amounts to a stepwise cycloaddition.

Alternatively, relaxation of $\mathbf{6}\left(\mathrm{T}_{1}\right)$ to the oxidopyrylium ground state $\mathbf{6}\left(\mathrm{S}_{0}\right)$ can occur (pathway (2)). The calculated absorption spectrum of $\mathbf{6}\left(\mathrm{S}_{0}\right)$ at $\lambda_{\max }=405 \mathrm{~nm}$ is well-matched with the emission spectrum of the reptile lamp (see Fig. 5, cyan bar; see Supplementary Table 5 and Fig. 4 for details), allowing it to undergo another photochemical reaction. After excitation to $\mathbf{6}\left(\mathrm{S}_{2}\right)$ a low lying conical intersection seam connecting all three singlet states $S_{2}, S_{1}$ and $S_{0}$ can be reached. The geometry leading to the formation of epoxy cyclopentenone $\mathbf{1 6}$ is marked as $S_{1} / S_{0}$-CoIn in Fig. 6b (see Supplementary Tables 6 and 33). From a more synthetic point of view, this process can be classified as a disrotatory electrocyclization in the excited state. From $6\left(\mathrm{~S}_{2}\right)$, the formation of intricarene (4) can also occur via the $\mathrm{T}_{1}$ state and via conical intersections among the singlet states.

In the presence of water, the vinyl epoxy cyclopentenone $\mathbf{1 6}$ is presumably highly unstable. We propose that it undergoes vinylogous nucleophilic substitution to afford the experimentally observed hydroxy cyclopentenone 11.

Our mechanism for the formation of the oxidopyrylium via homolytic bond cleavage in the excited state explains why the reaction can take place in the presence of water and why the acetate 13 failed to yield intricarene. The bond dissociation energy $(\mathrm{BDE})$ of methanol $\left(\mathrm{DH}_{298}=104.6 \pm 0.7 \mathrm{kcal} \mathrm{mol}^{-1}\right)$ is significantly lower than that of water $\left(\mathrm{DH}_{298}=118.82 \pm 0.07\right.$ $\left.\mathrm{kcal} \mathrm{mol}^{-1}\right)$. In contrast, homolytic cleavage of the acetate 13 would yield a highly unstable acetoxy radical (the BDE of acetic acid is $D_{298}=112 \pm 3 \mathrm{kcal} \mathrm{mol}^{-1}$ ) and is therefore less favourable than the homolytic cleavage of methyl ether 8 (ref. 28).

\section{Discussion}

Our work raises interesting questions regarding the classification of intricarene as a natural product. We define a natural product as 


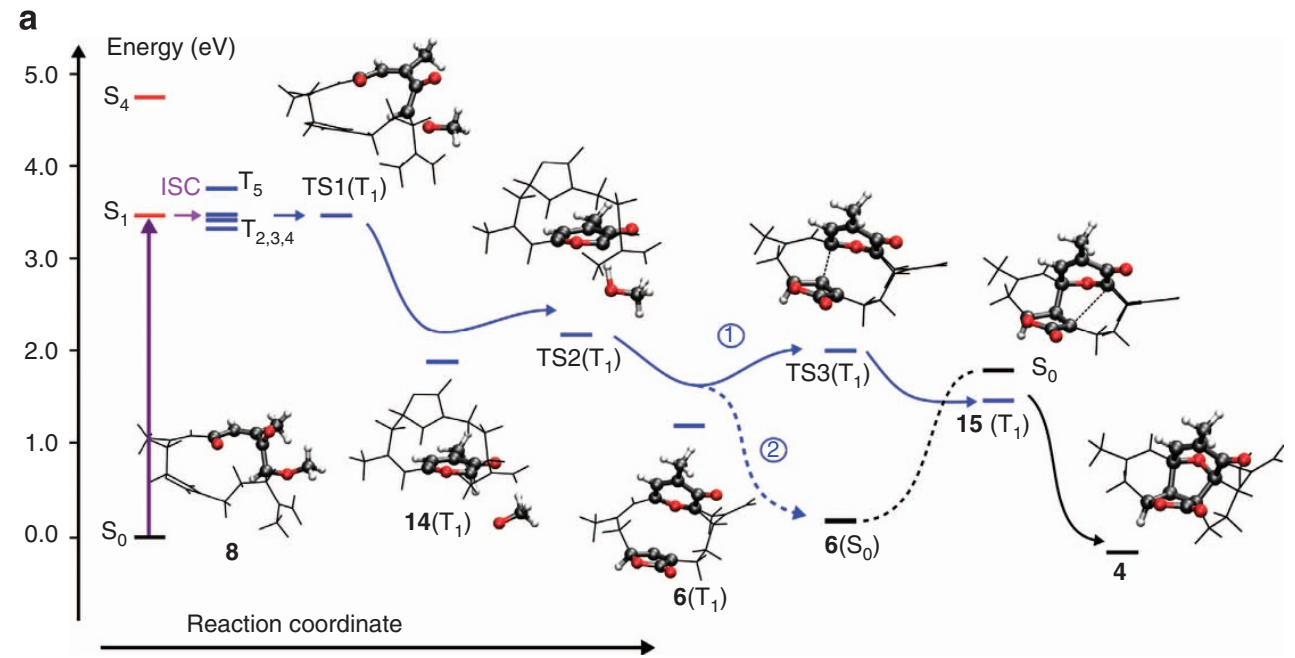

C

b

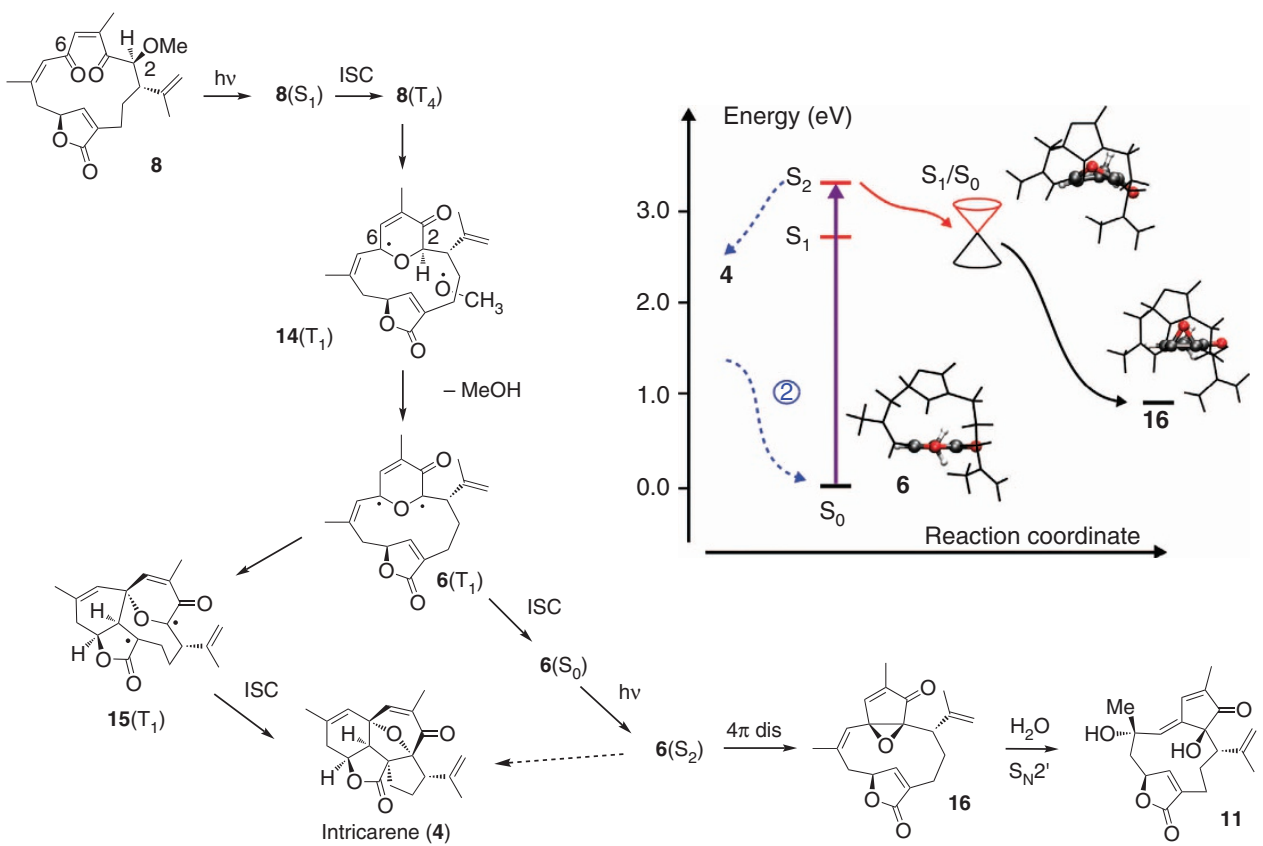

Figure 6 | Overview of all reactions steps for the proposed mechanism. (a) Calculated reaction path for photo-excited $\mathbf{8}$. Shown are the $\Delta G$ values (in $\mathrm{eV}$ ) for the isolated molecules. Excitation of $\mathbf{8}$ with UV-A light and sub-ps ISC to the degenerate T4 state and IC to the T1 state allows the formation of the intermediate diradical $\mathbf{1 4}\left(\mathrm{T}_{1}\right)$. Next, a hydrogen atom is transferred to afford the triplet diradical $\mathbf{6}\left(\mathrm{T}_{1}\right)$. This intermediate either undergoes a stepwise 1,3 dipolar cycloaddition, including an ISC, to afford $\mathbf{4}$ (pathway (1)), or relaxes to the ground state oxidopyrylium $\mathbf{6}\left(\mathrm{S}_{0}\right)$ ) (via pathway (2)).

(b) Oxidopyrylium $\mathbf{6}\left(\mathrm{S}_{0}\right)$ can undergo further photochemical reactions. After excitation with blue light to the S2 state, a S1/S0 conical intersection seam can lead to epoxy cyclopentenone $\mathbf{1 6}$ in a reaction that could be interpreted as a conrotatory $4 \pi$-electrocyclization. (c) Graphical representation of the reaction path. Vinylogous nucleophilic substitution with water affords $\mathbf{1 1}$ from 16.

a compound that is present within or in the immediate vicinity of the producing organism. As such, many compounds that are spontaneously formed from reactive intermediates, for instance via cascade reactions ${ }^{29-34}$, can indeed be classified as natural products. Our hypothetical starting material $O$-Methyl bipinnatin J (7), however, has been suspected to be an isolation artefact as methanolysis of bipinnatin J (1) and of kallolide (2) is a very facile process $^{18}$. In addition, while $\mathbf{4}$ could biosynthetically formed from 1 and $S$-adenosyl methionine by an $O$-methyl transferase, it is conspicuous that $\mathbf{4}$ is the only $\mathrm{C} 2$ methyl ether known in the entire furanocembranoid series ${ }^{35}$.

In the course of the isolation of intricarene, P. kallos was partially sun-dried (with indirect sunlight), frozen and lyophilized before extraction ${ }^{6}$. The dry organism was blended using a 1:1 mixture of dichloromethane and methanol. This suggests that the photochemical oxidation and cycloaddition could have taken place during workup. In contrast, clear sea water is known to only weakly attenuate UV-A/blue light ${ }^{36,37}$. Thus, at a depth of $25-28 \mathrm{~m}$, where the octocoral was collected, the intensity of incident UV-A and blue light is still well within the range of indirect sunlight at sea level and high enough to promote the proposed photochemistry over time (see Supplementary Fig. 2 and Supplementary Discussion (Irradiance in the natural habitat of the coral)). All things considered, we cannot decide whether intricarene is a genuine natural product according to the definition given above or an isolation artefact that was formed in the course of the isolation/purification procedure. This question could be settled by isolating intricarene under 
conditions that rigorously exclude air, intense light and methanol during workup.

In sum, our work provides a rationale for the formation of intricarene, which is based on photochemistry and overcomes the need to invoke a thermally challenging 1,3-dipolar cycloaddition. Our quantum chemical calculations provide detailed insights into the largely unexplored excited state chemistry of oxidopyrylium species. Finally, our results demonstrate that photochemical reactions, especially reaction cascades that proceed through a series of intermediates, need to be properly matched to the emission spectrum of the lamp used. As absorption spectra of putative intermediates can nowadays be calculated with relative ease, detailed information on the light source will greatly facilitate the planning and execution of photochemical reactions.

\section{References}

1. Dewick, P. M. Medicinal Natural Products (John Wiley \& Sons, 2009).

2. Rodríguez, A. D. \& Shi, J.-G. The first cembrane-pseudopterane cycloisomerization. J. Org. Chem. 63, 420-421 (1998).

3. Look, S. A., Burch, M. T., Fenical, W., Zheng, Q. \& Clardy, J. Kallolide A, a new antiinflammatory diterpenoid, and related lactones from the Caribbean octocoral Pseudopterogorgia kallos (Bielschowsky). J. Org.Chem. 50, 5741-5746 (1985).

4. Weinstabl, H., Gaich, T. \& Mulzer, J. Application of the Rodriguez-Pattenden photo-ring contraction: total synthesis and configurational reassignment of 11-Gorgiacerol and 11-Epigorgiacerol. Org. Lett. 14, 2834-2837 (2012).

5. Marrero, J. et al. Bielschowskysin, a Gorgonian-derived biologically active diterpene with an unprecedented carbon skeleton. Org. Lett. 6, 1661-1664 (2004)

6. Marrero, J., Rodríguez, A. D. \& Barnes, C. L. Intricarene, an unprecedented trispiropentacyclic diterpene from the caribbean sea plume Pseudopterogorgia kallos. Org. Lett. 7, 1877-1880 (2005).

7. Roethle, P. A., Hernandez, P. T. \& Trauner, D. Exploring biosynthetic relationships among furanocembranoids: synthesis of $(-)$-bipinnatin J, (+)-intricarene, (+)-rubifolide, and (+)-isoepilophodione B. Org. Lett. 8, 5901-5904 (2006).

8. Tang, B., Bray, C. D. \& Pattenden, G. A biomimetic total synthesis of (+)-intricarene. Tetrahedron Lett. 47, 6401-6404 (2006).

9. Doroh, B. \& Sulikowski, G. A. Progress toward the total synthesis of bielschowskysin: a stereoselective $[2+2]$ photocycloaddition. Org. Lett. 8, 903-906 (2006).

10. Miao, R., Gramani, S. \& Lear, M. Stereocontrolled entry to the tricyclo[3.3.0] oxoheptane Core of bielschowskysin by a $[2+2]$ cycloaddition of an allene-butenolide. Tetrahedron Lett. 50, 1731-1733 (2009).

11. Nicolaou, K. C., Adsool, V. \& Hale, C. An expedient synthesis of a functionalized core structure of bielschowskysin. Angew. Chem. Int. Ed. 50, 5149-5152 (2011).

12. Farcet, J.-B., Himmelbauer, M. \& Mulzer, J. A non-photochemical approach to the bicyclo[3.2.0]heptane core of bielschowskysin. Org. Lett. 14, 2195-2197 (2012).

13. Himmelbauer, M., Farcet, J.-B., Gagnepain, J. \& Mulzer, J. A palladiumcatalyzed carbo-oxygenation: the bielschowskysin case. Org. Lett. 15 3098-3101 (2013).

14. Townsend, S. D. \& Sulikowski, G. A. Progress toward the total synthesis of bielschowskysin. Org. Lett. 15, 5096-5098 (2013).

15. Yang, E. G., Sekar, K. \& Lear, M. J. A macrolactonisation approach to the cembrane carbocycle of bielschowskysin. Tetrahedron Lett. 54, 4406-4408 (2013).

16. Wang, S. C. \& Tantillo, D. J. Theoretical studies on synthetic and biosynthetic oxidopyrylium - alkene cycloadditions: pericyclic pathways to intricarene. J. Org. Chem. 73, 1516-1523 (2008).

17. Roethle, P. A. \& Trauner, D. Expedient synthesis of ( \pm )-bipinnatin j. Org. Lett. 8, 345-347 (2006).

18. Rodríguez, A. D., Shi, J.-G. \& Shi, Y.-P. Isolation, structural characterization, and synthesis of a naturally occurring bisfuranopseudopterane ether: biskallolide A. Evidence for a carbocation intermediate during the facile conversion of kallolide A and isokallolide A into various solvolysis products. J. Org. Chem. 65, 3192-3199 (2000).

19. Ambrosio, M. D., Guerriero, A. \& Pietra, F. Novel cembranolides (coralloidolide D and E) and a 3,7-cyclized cembranolide (coralloidolide C) from the mediterranean coral Alcyonium coralloides. Helv. Chim. Acta 72, 1590-1596 (1989).

20. Williams, D., Andersen, R. J., Van Duyne, G. D. \& Clardy, J. Cembrane and pseudopterane diterpenes from the soft coral Gersemia rubiformis. J. Org. Chem. 52, 332-335 (1987).

21. Olivucci, M. Computational Photochemistry (Elsevier B.V., 2005).

22. Klessinger, M. \& Michl, J. Excited States and Photochemistry of Organic Molecules (VHC Publisher Inc, 1995).

23. Marian, C. M. Spin-orbit coupling and intersystem crossing in molecules. WIREs Comput. Mol. Sci. 2, 187-203 (2012).

24. Griesbeck, A. G., Abe, M. \& Bondock, S. Selectivity control in electron spin inversion processes: regio- and stereochemistry of paternò-büchi photocycloadditions as a powerful tool for mapping intersystem crossing processes. Acc. Chem. Res. 37, 919-928 (2004).

25. Serrano-Pérez, J. J., Merchán, M. \& Serrano-Andrés, L. Photoreactivity of furocoumarins and DNA in PUVA therapy: formation of psoralen-thymine adducts. J. Phys. Chem. B 112, 14002-14010 (2008).

26. Richter, M., Marquetand, P., González-Vázquez, J., Sola, I. \& González, L. Femtosecond intersystem crossing in the DNA nucleobase cytosine. J. Phys. Chem. Lett. 3, 3090-3095 (2012).

27. Albini, A. \& Fagnoni, M. Photochemically-Generated Intermediates in Synthesis 74-75 and 145-147 (John Wiley \& Sons, 2013).

28. Blanksby, S. J. \& Ellison, G. B. Bond dissociation energies of organic molecules. Acc. Chem. Res. 36, 255-263 (2003).

29. Grondal, C., Jeanty, M. \& Enders, D. Organocatalytic cascade reactions as a new tool in total synthesis. Nat. Chem. 2, 167-178 (2010).

30. Nicolaou, K. C. \& Chen, J. S. The art of total synthesis through cascade reactions. Chem. Soc. Rev. 38, 2993-3009 (2009).

31. Volgraf, M. et al. Biomimetic Synthesis of the IDO inhibitors exiguamine A and B. Nat. Chem. Bio. 4, 535-537 (2008).

32. Strych, S. \& Trauner, D. Biomimetic synthesis of santalin A, B and santarubin A, B, the major colorants of red sandalwood. Angew. Chem. Int. Ed. 52, 9509-9512 (2013).

33. Sofiyev, V., Navarro, G. \& Trauner, D. Biomimetic Synthesis of the Shimalactones. Org. Lett. 10, 149-152 (2008).

34. Beaudry, C. M. \& Trauner, D. Total Synthesis of ( - )-SNF4435 C and (+)-SNF4435 D. Org. Lett. 7, 4475-4477 (2005).

35. Roethle, P. A. \& Trauner, D. The chemistry of marine furanocembranoids, pseudopteranes, gersolanes, and related natural products. Nat. Prod. Rep. 25, 298-317 (2008)

36. Armstrong, F. A. J. \& Boalch, G. T. The ultra-violet absorption of sea water. J. Mar. Biol. Ass. UK 41, 591-597 (1961).

37. Hargreaves, B. R. Helbling, E. W. \& Zagarese, H. E. (eds) Comprehensive Series in Photochemical and Photobiological Sciences (Royal Society of Chemistry, 2003).

\section{Acknowledgements}

We thank the Deutsche Forschungsgemeinschaft (SFB749) for financial support.

\section{Author contributions}

D.S. and T.J.K. performed the synthetic work and analysed the obtained products. D.T. devised the study. P.K. performed the quantum chemical calculations. E.R. devised and accompanied the photophysical aspects of the study. D.T., P.K., E.R. and R.dV.-R. wrote the manuscript.

\section{Additional information}

Accession codes: The X-ray crystallographic coordinates for structures reported in this article have been deposited at the Cambridge Crystallographic Data Centre (CCDC), under deposition numbers CCDC 1026998 and 1026997. These data can be obtained free of charge from The Cambridge Crystallographic Data Centre via http:// www.ccdc.cam.ac.uk/data_request/cif

Supplementary Information accompanies this paper at http://www.nature.com/ naturecommunications

Competing financial interests: The authors declare no competing financial interests.

Reprints and permission information is available online at http://npg.nature.com/ reprintsandpermissions/

How to cite this article: Stichnoth, D. et al. Photochemical formation of intricarene. Nat Commun. 5:5597 doi: 10.1038/ncomms6597 (2014). 